\title{
Publisher's Note: Gating Classical Information Flow via Equilibrium Quantum Phase Transitions [Phys. Rev. Lett. 118, 147203 (2017)]
}

Leonardo Banchi, Joaquín Fernández-Rossier, Cyrus F. Hirjibehedin, and Sougato Bose

(Received 10 April 2017; published 17 April 2017)

DOI: 10.1103/PhysRevLett.118.169901

This paper was published online on 5 April 2017 with an error in the author list. The second author's name should read as "Joaquín Fernández-Rossier." The author name has been corrected as of 7 April 2017. The author name is correct in the printed version of the journal. 